\title{
Reviewing Spectroscopic Techniques: A New Weapon for Monitoring Corona Virus
}

\author{
Jagriti Chatterjee $^{1} \mid$ Subhojyoti Chatterjee ${ }^{2}$ \\ ${ }^{1}$ Department of Bioinformatics, Karunya Institute of Technology and Sciences, Coimbatore, Tamilnadu, India \\ ${ }^{2}$ School of Biotechnology, Amrita Vishwa Vidhyapeetham, Kollam, Kerala, India.
}

To Cite this Article

Jagriti Chatterjee and Subhojyoti Chatterjee, "Reviewing Spectroscopic Techniques: A New Weapon for Monitoring Corona Virus", International Journal for Modern Trends in Science and Technology, 6(8): 44-50, 2020.

\section{Article Info}

Received on 06-June-2020, Revised on 28-June-2020, Accepted on 25-July-2020, Published on 29-July-2020.

\section{ABSTRACT}

Chemical, structural, and physical properties are parameterized and comprehended in the molecular properties of a molecule. They are therefore calculable and can be categorized as molecular energetics, spectral quantities, and properties that are not physical observables (for example, aromaticity, isoelectronic and bond order), i.e., measurable and non-measurable properties. Different aspects of theory and experiments give more insightful information of biologically living complex macromolecules. For example, the structure of nucleic acids and proteins (like the CoV envelope (E) protein)are measured by $X$-ray crystallography and NMR techniques, etc., in order to understand their properties and functionalities. More detailed structural information of these biomolecules can further be captured through techniques such as molecular spectroscopy. For example, X-ray photoemission spectroscopy captures insight into the molecular interactions and bonding nature of the biomolecules (such as envoloped RNA coronaviruses). These biologically important spectroscopic techniques have been discussed in this mini review report.

KEYWORDS: Coronavirus, CoV envelop protein, Atomic spectroscopy, Fluorescence spectroscopy, Infrared spectroscopy, Nuclear magnetic resonance spectroscopy, Ultravioletand visible light spectroscopy.

\section{INTRODUCTION}

Spectroscopy may be defined as "the analysis of the relationship between matter and electromagnetic radiation" (Yadav, 2005). The spectroscopic approaches compose of the largest and the most important class of procedure which is applied in analytical chemistry. The spectroscopic approach furnishes with qualitative and quantitative details (Sharma, 2017). Spectroscopy and its applications form a significant part of modern chemistry and physics (Seybold et al., 1987). Whence the word spectroscopy comes, it signifies the vigilance of images, but the new subject involves the relationship of electromagnetic radiations with the matter. The most important result of such interaction is that "energy is absorbed or emitted by matter in the distinct amount called quanta"(Yadav, 2005; Seybold et al., 1987). The absorption or emission techniques are known throughout the electromagnetic spectrum ranging from $\gamma$-region to the radio wave region. The advantages of spectroscopy include (i) The substance is required in very small quantities; (ii) Spectroscopic methods are easier and faster to do than most chemical radiations or tests, and; (iii) The methods are extremely sensitive, and the information obtained is highly reliable(Seybold et al., 1987). 
A celebrated example demonstrating advantage of spectroscopic techniques can be seen in today's pandemic situation-COVID-19. To explain further, scientists can use vibrational spectroscopic techniques to study the structure of the Corona Virus, obviously maintaining the safety norms (Khan et al., 2020). Complex biological samples collected from positive coronavirus(SARS-CoV-2) patients (i.e., urine, blood, sputum) can be analyzed with great efficiency, using spectroscopic techniques like FT-IR, Raman and Proton $\left({ }^{1} \mathrm{H}\right)$ nuclear magnetic resonance (NMR) spectroscopy, an example of which is illustrated in Fig. 1 (Khan et al., 2020).

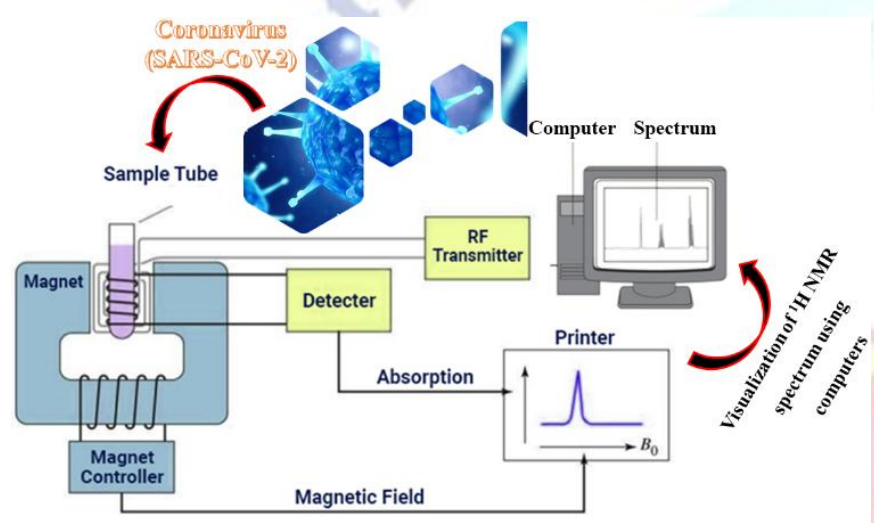

Fig. 1: Representation of ${ }^{1} \mathrm{H}$ NMR spectroscopy for analyzing coronavirus (SARS-CoV-2 / COVID19) samples (Khan et al., 2020).

Spectroscopy can be broadly classified as two types (Slater, 1929), namely: (a) Atomic spectroscopy - It involves with the relationship of electromagneticradiation (EMR, described later) with atoms which are mostly in their lowest energy state also known as the ground state (Bhagwan, 2005).Normally, the existence of monoatomic substances is in the gaseous state and can absorb electromagnetic radiation, resulting in electrons transitions from one electronic energy level to another(Bhagwan, 2005). The absorption of electromagnetic radiation can take place only if the photon has an energy which is similar to the change in energy between two quantized energy levels (Eq. 1), i.e.,

$$
\Delta E=h \vartheta
$$

where $\Delta E$ is equal to the change in energy between two quantum levels and $\vartheta$ is the frequency of photon which can result in the electronic excitation. (b) Molecular spectroscopy - It takes care of the interaction between the electromagnetic radiation and molecules. In addition to electronic transitions, it also results in the changeover between rotational and vibrational energy stages which in turn complicates the spectra of molecules much more than the atoms(Schrodinger, 1926). Molecular spectra which has its stretch from the visible via infrared to the microwave region, has been of current interest in molecular spectroscopy mainly because the number of known molecules is very huge in comparison to the free atoms. The interest also lies due to the present $\mathbf{x}$-ray structural elucidation of coronavirus (COVID-19 / SARS-CoV-2)where they have found that it is enveloped by a lipid bilayer membrane and the spike glycoprotein helps in the anchoring inside the host cell(Khan et al., 2020). Furthermore, the genomic RNA along with nucleocapsid protein mimics the host cell properties thereby helping in multiplication and spreading the disease from one host to another, as represented in Fig. 2.

What is electromagnetic radiation (EMR)?(Zumdahl et al., 2003) It is a type of energy that is disseminated through space at a huge velocity. Electromagnetic radiation is supposed to have a dual nature as it showcases both wave and particle characteristics. This duality is not only restricted to the visible region of the electromagnetic spectrum (EMS) but also can be explained for the entire portion of electromagnetic radiation. Exploration of the wave properties of electromagnetic radiation (Fig.3), shows that it is a varied electrical and magnetic field identified in space. Hence, an electromagnetic wave has both an electronic and a magnetic characteristic. These two properties vibrate in planes perpendicular to each other and perpendicular to the direction of propagation of the radiation(Zumdahl et al., 2003).

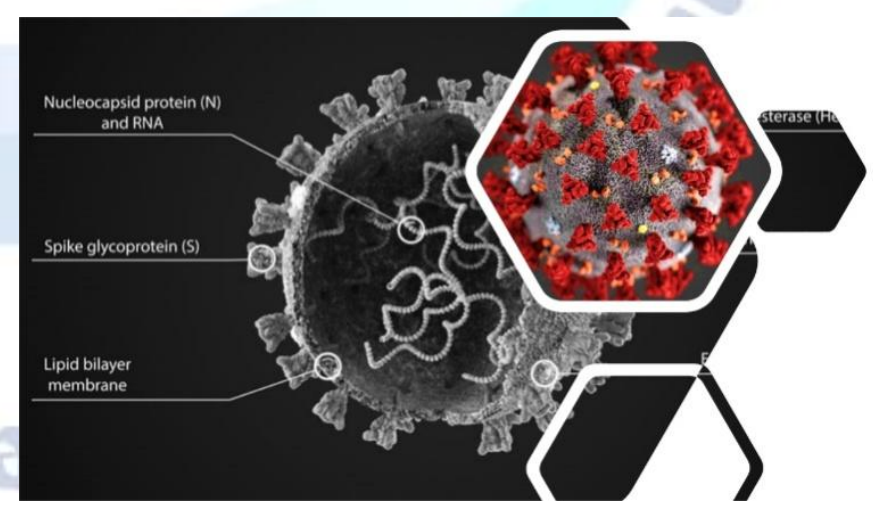

Fig. 2: X-rays coronavirus protein structure (Khan et al., 2020).

What is electromagnetic spectrum (EMS)? It is defined as the electric range over which electromagnetic radiation exists (in Fig.4). This oscillates at 90 degrees to the direction of propagation and thus impacting a wave motionto 
the radiation. The wavelength of EMR varies from a few angstroms to several meters. The unitsused to describe the wavelengths are as follows:

$\AA$ A $=$ Angstrom $=10^{-10}$ centimeter $(\mathrm{cm})=10^{-4}$ micrometer $(\mathrm{mm})$

$\mathbf{n m}=$ nanometer $=10^{-9}$ meter $(\mathrm{m})=10 \AA=10^{-3} \mu \mathrm{m}$ $\boldsymbol{\mu m}=$ micrometer $=10^{-6}=10^{4} \AA$

$\boldsymbol{\Lambda}=$ unit for $\mathrm{UV}$ and visible region $=\mathrm{nm}$

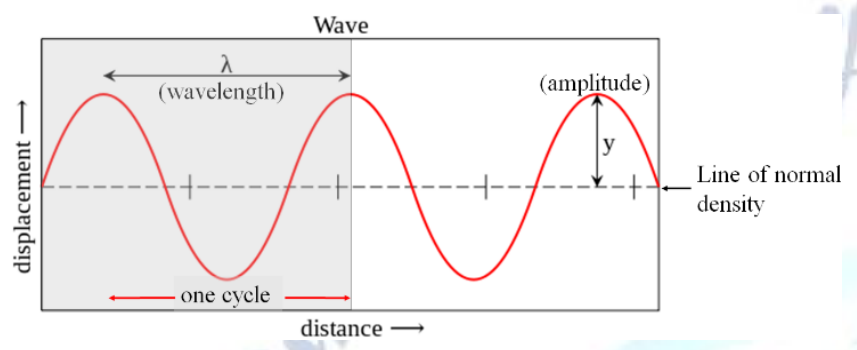

Fig.3: Representation of an electromagnetic wave(Maxwell, 1865).

\section{TyPES OF SPECTROSCOPY}

The areas of the electromagnetic spectrum and their associated processes are extensively used for daily analytical business and research in biological field. It is easy to tackle with the appropriate rules in relation to the absorption of light with that of the portion of the electromagnetic spectrum for which these laws were made(Sharma, 2017). Although there are different types of spectroscopy, however for chemical analysis, the most common types used are:

\section{A. Ultraviolet and visible light spectroscopy}

Principles: The Beer-Lambert law is an amalgamation of two laws each taking care of the absorption of light in relation to the concentration of the absorber and the path length or thickness of the layer separately. Given that an absorbingsubstance is partially transparent and will transfer

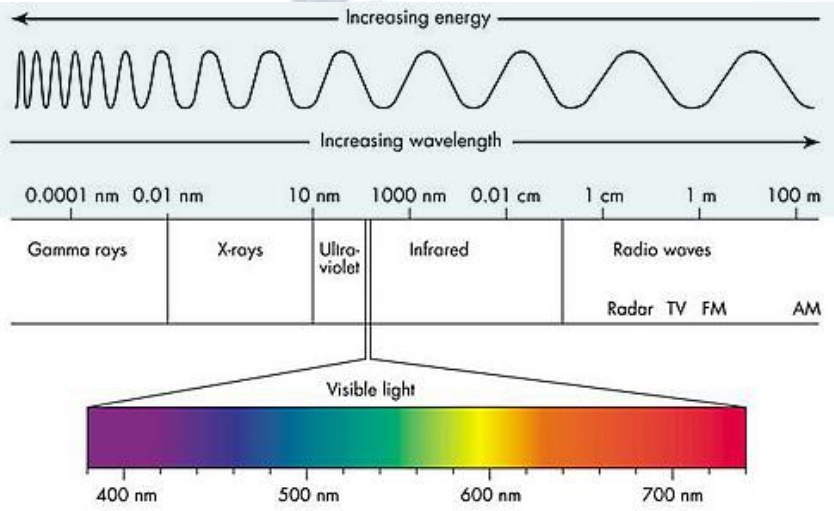

Fig. 4: The electromagnetic spectrum(Maxwell, 1865). a part of the radiation incident upon it, as shown inFig.5. The ratio of the intensities of transmitted and incident light results in the transmittance $\mathrm{T}$, expressed as Eq. 2

wherein,

$$
T=I / I_{0}
$$

$\mathbf{I}_{\mathbf{0}}=$ is the intensity of incident radiation

$\mathbf{I}=$ is the intensity of transmitted radiation

The value of $\mathrm{T}$ as $100 \%$ depicts that the transparent substance has no radiation absorbed, whereas a zero value of $T$ shows that the substance is an opaque one which in turn effectthe complete absorption. For exact values, we can explain the absorbance (A) or extinction (E) which is represented by logarithm (to base 10) of the reciprocal of the transmittance (Eq. 3).

$$
A=E=\log _{10}(I / T)=\log _{10}\left(I_{0} / I\right)
$$

Absorbance which is also known as the optical density (O.D), has its logarithm value (unitless) and ranging from $\mathbf{O} \equiv(\mathbf{1 0 0 \%} \mathbf{T})$ to $\mathbf{a} \equiv(\mathbf{0} \mathbf{T})$.

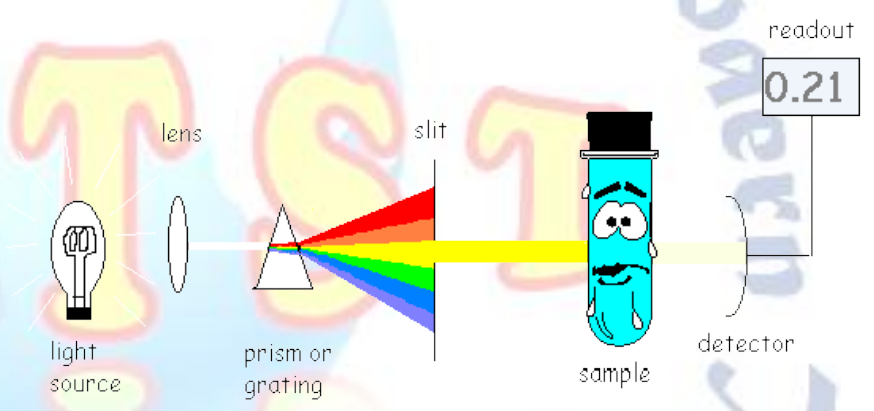

Fig. 5: Principle of Beer-Lambert Law (De Caro, 2015).

Hence, the Beer-Lambert Law(De Caro, 2015) states that "absorbance is proportional to both the concentration of absorbent and thickness of the layer" (Eq. 4).

$$
A=\varepsilon_{\lambda} C L
$$

wherein,

$\boldsymbol{\varepsilon}_{\lambda}=$ Molar absorbance coefficient for the absorber wavelength $\lambda$

$\boldsymbol{C}=$ Concentration of the absorbing solution

$\boldsymbol{L}=$ Path length through the solution

Instrumentation:The materials used are of optical instruments which depend on the wavelength, in the UV-region. Prisms, gratings, reflectors, and cuvettesmade up of silica are used. The radiation source is usually Argon, Xeon lamp $(\mathbf{\lambda}=\mathbf{1 5 0}-\mathbf{6 0 0} \mathbf{n m})$ are used to produce UV-rays. Tungsten filament and halogen lamps are used to produce the radiation in the visible region. The monochromator used in graters is made of silica and quartz. The cuvettes are usually lithium fluoride and fused silica are employed in the UV 
region. Detectors used in a photocell that convert the light energy into electrical energy are Photocathode, Dyanode, and Anode. Lastly, the function of a recorder is to measure the variation in excitement at a predetermined wavelength with time (De Caro, 2015).

Applications:The usage of UV-Vis spectroscopy includes (a) quantitative analysis that can be carried out in the UV/visible region to predict particular classes of compounds both in the pure state and in biological mixtures; (b) The technique also has the capability to express chemical structures and intermediates that take place in a system and (c) In the quantitative analysis of certain chromophores(De Caro, 2015).

\section{B. X-ray spectroscopy}

Principles:Where, $\gamma$-rays are of nuclear origin, $\mathrm{X}$-rays arise from displacement of inner extranuclear electrons. The electrons with principle quantum numbers 1,2 , and 3 in an atom can be imagined occupying shells $-\mathrm{K}, \mathrm{L}$, and $\mathrm{M}$, respectively. X-rays can be absorbed by matter and this gives rise to X-ray absorption spectra. The rules applying to the relationship between an incident beam of monochromatic radiation $\left(\boldsymbol{I}_{\boldsymbol{o}}\right)$ and the transmitted portion $\boldsymbol{I}$, are like the Beer-Lambert case. If $\mu$ is the linear absorption coefficient of the absorbing material, then (Eq. 5),

$$
I=I_{0} e^{-\mu x}
$$

where, $\mathbf{x}=$ the thickness of the absorber.

Instrumentation: A suitable $\mathrm{x}$-ray source is required that can be focused into the specimen chamber, where the substance under test is excited by the incident beam. A monochromator is required to disperse the fluorescent radiation and finally, a suitable detector and data processing facilities are needed. X-ray spectrometers require a more rigorous approach to the incorporation of

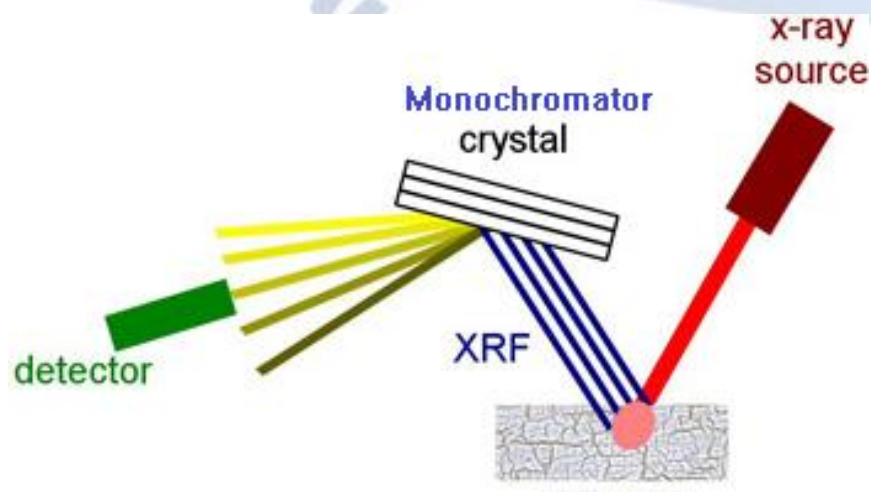

Sample

Fig.6: X-ray fluorescence analysis, i.e., depression of fluorescent $\mathrm{x}$-ray detected at a various angle(Dingle, 1935). safety features, but the essential requirements of source monochromator and detector are the same, as can be seen in Fig. 6 .

Applications:The technique has wide applications in forensic science and environmental pollution studies because many useful elements can be detected and concentration measured by the technique is essential for uncovering the trace elements, for example in fertilizers.

\section{Nuclear magnetic resonance (NMR) spectroscopy}

Principles: Most studies involve the use of ${ }^{1} \mathrm{H}$, but ${ }^{13} \mathrm{C},{ }^{15} \mathrm{~N}$, and ${ }^{31} \mathrm{P}$ isotopes are also exploited in biochemical studies. The vibration conditions in NMR are supplied in an external magnetic field of numerous hundred count with absorptions taking place in the region of radio waves of $40 \mathrm{MHz}$ frequency for the vibration of the ${ }^{1} \mathrm{H}$ nucleus (as can be seen in Fig.7, an example for ethyl alcohol). The actual field scrutinized is small in comparison to the total field applied and the radio frequencies absorbed are particularly stated on such spectra. NMR spectra are of much importance in illustrating chemical structures. Both qualitative and quantitative information may be accessed, and hyperfine splitting produces information related to the neighborhood of the nucleus(Khan et al., 2020; Dingle, 1935).
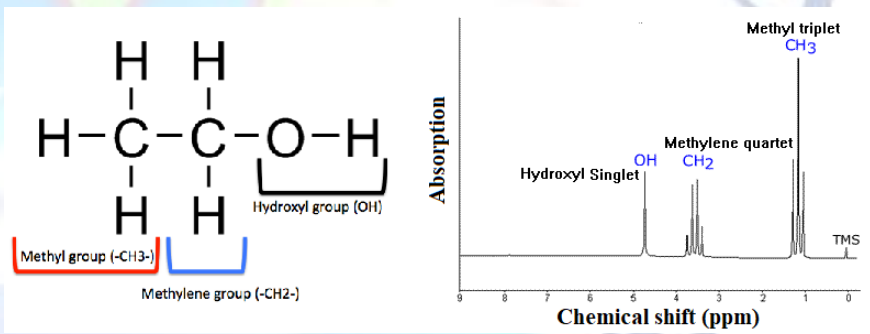

Fig.7: ${ }^{1} \mathrm{H}$ NMR spectrum of ethyl alcohol(Dingle, 1935).

Instrumentation: The instrumentation of NMR spectroscopy involves nine major parts (see Fig. 1). There is a Sample Holder which is made up of glass. The Magnetic Coils produce magnetic field whenever there is a flow of current. Also, there is a Permanent Magnet to produce homogenous magnetic field within the range of $60-100 \mathrm{MHz}$. The Sweep Generator involves itself in modifying the magnetic field generated. Radiofrequency and Radiofrequency Transmitter are responsible for detecting receiver radio frequencies and produce short but strong pulse of radio waves, respectively. $\mathrm{RF}$ detector detects the unabsorbed radio frequencies. The NMR of these unabsorbed radio 
frequencies are noted by the Recorder. Finally, there is the Readout system attached to a computer to record the NMR data.

Applications: (a) The illustration of molecular structure, conformational changes, and particular kind of active analysis are the main applications of NMR in the biological filed, with special mention for coronavirus (SARS-CoV-2). Mostly suchwork isdone in solution environment and to reduce solvent effects thedeuterated solvent isused; (b) The exploitation of thistechnique in drug metabolism studies is of great importance, specifically when connected with infrared and $\mathrm{x}$-ray diffraction data, which can later be used in molecular modelling techniques using sophisticated computer applications to explain drug action (like identifying miRNA target sites for probableCOVID-19 drug binding interaction); (c) The use of NMR in tissue and whole animal studies is developing rapidly, with some physiological explanation that can be extended to the human studies. ATP metabolism in healthy and unhealthy individuals and changes during exercise can also be measured; (d) One of the body scanners used for the location of tumors is based on NMR using ${ }^{1} \mathrm{H}$ resonance in water which appears to be in greater concentration in rapidly dividing cells in a tumor mass(Dingle, 1935).

\section{Infrared spectroscopy}

Principles:The process is established upon the fact that achemical substance spectacles distinct selective absorption in the infra-red region. After absorption of IR radiation, the molecules of a chemical substance resonate at many rates of vibrations, leading to close-packed absorption bands, known as IR absorption spectrum which may stretch over a wide wavelength band. Band positions in an infrared spectrum may be clarifiedeasily by the wavenumber $v$, where the unit is $\mathrm{cm}^{-1}$, as can be seen in Fig. 8, an example for phenacetin. The affiliation between wave number $v$ and wavelength $\lambda$ and frequency $\phi$ is as follows (Eq. 6)

$$
v=1 / \lambda, \text { since, } \phi=c \lambda
$$

where ' $c$ ' is defined as the velocity of light. The infrared radiation invokes broadly to that portion of the electromagnetic spectrum which lies in between the visible and microwave portion.

Instrumentation:The instrument involves fixed and rotating mirrors that split the incident beam into two. The beams are recombined after passage through the sample but as the two path lengths are different, interference patterns arise that may be

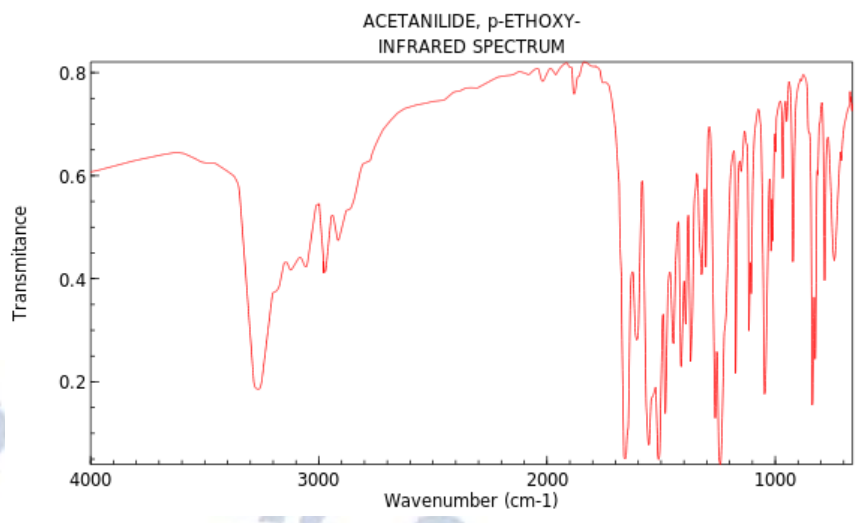

Fig.8: FT-IR spectrum of phenacetin. Bands at the appropriate frequency $\left(\mathrm{cm}^{-1}\right)$ are shown(Slater, 1929).

analyzed by Fourier Transform methods. TheBeer-Lambert Law applies in all cases except for complex mixtures, where more complicated mathematical procedures are required.

Applications:The application of infrared and Raman spectroscopy is mainly in the field of biochemical research for intermediate-sized molecules such as drugs, metabolic intermediates, and substrates(Dingle, 1935).

\section{E. Fluorescence spectroscopy}

Principles: Fluorescence is an emission process, wherein, the energy transfers from a higher to lower state. The molecule involved being measured by the exposure of this emitted radiation rather than the absorption. For the transfer from higher to lower states, an earlier excitation event must have taken place. This earlier event is due to the impact of energy by the absorption of electromagnetic radiation. The wavelength of absorbed radiation is supposed to be of lower values than the emitted wavelength, as can be seen in Fig.9.

Fluorescence spectra gives competence and are generally independent of the exciting wavelength.

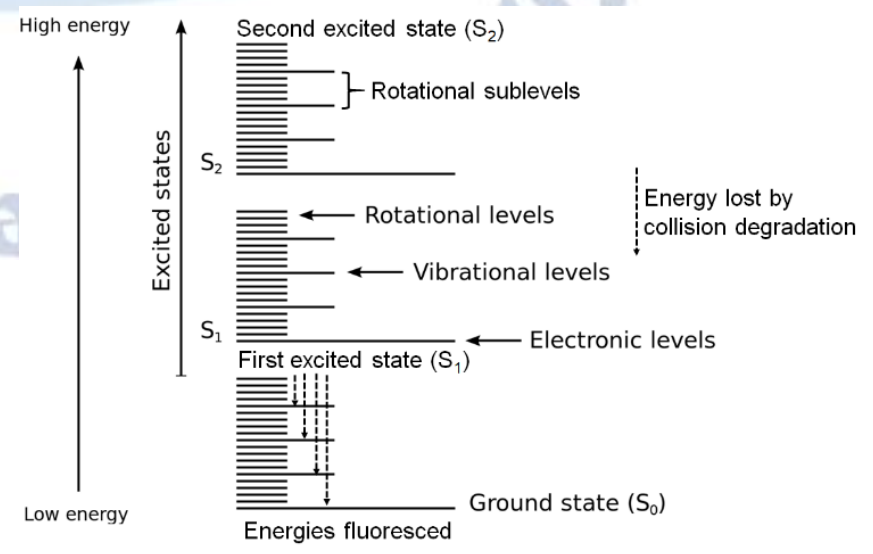

Figure 9: Energy levels and transitions of electrons(Lakowicz, 2006). 
At low concentrations, the magnitude offluorescence $\left(\boldsymbol{I}_{\boldsymbol{f}}\right)$ is correlated to the magnitude of the incident radiation (Io), and is given by (Eq. 7)

$$
I_{f}=I_{0} \varepsilon_{\lambda} c d Q
$$

where,

$\mathbf{c}=$ is the concentration of the fluorescing solution

$\mathbf{d}=$ is the light path in fluorescing solution

$\varepsilon=$ is the molar extinction coefficient for absorbing material at wavelength $\lambda$

Instrumentation: As stated above, the electrons required for spectrofluorometer were simpler than those required for absorptionspectrophotometers. The same is true fortheoptics. Two monochromators may be employed, the first $\left(\mathrm{M}_{1}\right)$ for choosing the excitation wavelength. Fluorescence emission takes place in all possible directions and one perpendicular direction is chosen and the monochromators $\left(\mathrm{M}_{2}\right)$ are used for the evaluation of the fluorescence spectrum. The radiation source is supposed to be either a mercury

lamp or a Xeon arc, with excitation wavelengths in the visible region. The detector is usually a sensitive photocell, as shown in Fig. 10.

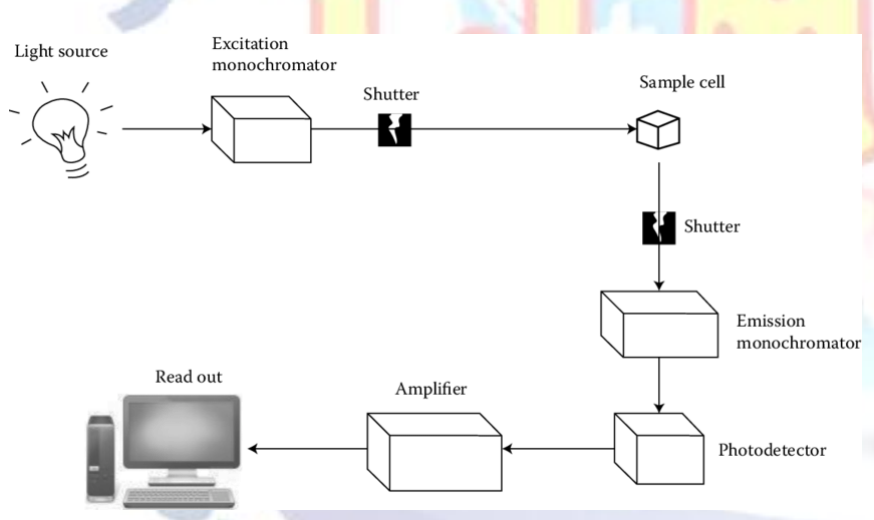

Fig.10: The basic component of a spectrofluorometer (Lakowicz, 2006).

Applications: (a) The major application of fluorimetry in biochemistry is in the qualitative prediction of materials present in very low concentration for absorption spectrophotometry. Assay of vitamin $\mathrm{B}_{1}$ in foodstuff, NADH, hormones, drugs, pesticides, carcinogens, chlorophyll, cholesterol, porphyrins, and some metal ions show the range.(b) Use in enzyme assay and kinetic analysis; (c) Also used in the determination of protein structures; (d) In membrane structure; (e) It is helpful in the energy transfer studies; and (f) It is also most importantly used in fluorescence immunoassay techniques(Lakowicz, 2006).

\section{F. Atomic absorption spectroscopy}

Principles:This techniquedetermines the amount of a specific element that may be presentin each complex sample. The light emission process is calculated by emission flame spectrophotometry and absorption by atomic absorption flame spectrophotometry.

\section{Instrumentation}

(atomic emission spectrophotometry):The nebulizers used are usually of the type that comprises passing a stream of air over a capillary tube with their other end dipping into the solution under test. Droplets are formed in various sizes and the larger ones may be allowed to be settled out in a cloud chamber. This step is required because the larger drops tend out to remain in the hottest part of the flame. This flame is long enough to indirectly inject into systems which constitutes of volatilized combustion of air and natural gas. The instrument is provided with a temperature of $1500^{\circ}$ which is sufficient for sample determination, as can be seen in Fig. 11.

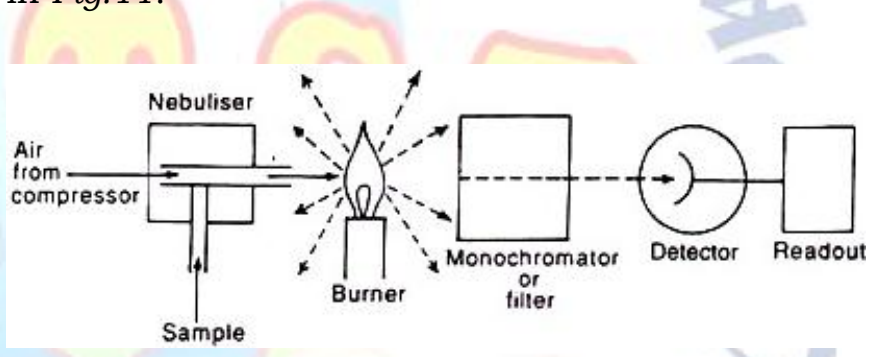

Fig.11: The basic component of a spectrofluorometer(Lakowicz, 2006).

Applications: This procedure is extensively used in clinical laboratories for the prediction of metals in body fluids. These determinations aid diagnosis and are valuable in monitoringmany therapeutic regimens. In physiological and pharmacological research, metals like sodium, potassium, calcium, lead, iron, and mercury needs to be previously extracted from the biological source and this helps in achieving the same. The methods are extensively used in element prediction in soil and plant materials and once the suitable ashing procedure is completed, it may be used for predicting metals in macromolecules, organelles, cells, and tissues(Lakowicz, 2006).

\section{Conclusions}

\section{The key conclusions are:}

$>$ Spectroscopy is used to study the molecular vibrations.

$>$ It is also used to explore both polar and non-polar interaction and to study both 
structural and conformational features of biomolecules having potential drug likeness property.

\section{A more specific role of spectroscopy includes:}

> The Ultra-Violet technique, which has the capability to express chemical structures and its intermediates that are involved in a kinematic reaction.

$>$ The $\mathbf{X}$-ray spectroscopy uncovers the essential trace elements, thereby, finding its applications in DNA fingerprinting, an important aspect of forensic science.

> Nuclear Magnetic Resonance (NMR) find its applications by differentiating the chemical environment of biomolecular isomers involved in different disease conditions like, cancer and SARS-CoV-2.

> Infrared (IR) spectroscopy elucidates structural and functional properties of intermediate sized molecules such as, chemical substrates, biomolecular isomeric drugs and intermediary metabolites.

> The resonance Raman spectroscopy (RR) is used to study the molecular aspects of chromatophores in hemoglobin's, flavonoids, carotenoids, and chlorophyll as well as in substrate binding sites in enzymes and metalloproteins.

> Fluorescence spectroscopy can be implied in treating cancers by predicting the biomarkers from bioluminescent organisms.

Hence, to conclude, spectroscopy gives amore detailed structural information ofbiomolecules by understanding their properties and functionalities. This opens new door for strucure based drug desiging, targeting deadly diseases like coronavirus (SARS-CoV-2), MERS, Flu,cancer, Alzheimers and diabetes.
- NADH - Nicotinamide Adenine Dinucleotide

- RR - Resonance Raman Spectroscopy

\section{ACKNOWLEDGMENT}

The authors would like to acknowledge Dr. Pratima Khandelwal, H.O.D Department of Biotechnology, Brindavan College for the insightful discussion on various spectroscopic techniques that helped in shaping the manuscript.

\section{REFERENCES}

[1] Yadav, L.D.S. (2005). Introduction to Spectroscopy (Spectrometry), In: Organic Spectroscopy.Springer, pp. $1-6$.

[2] Sharma, Ramesh K. (2017). Various Spectroscopic Techniques, In: Environmental Pollution Monitoring, Modeling and Control. Studium Press LLC, U.S.A., pp. 181-207.

[3] Seybold, P. G.; May, M.; Bagal, U. A. (1987). Molecular structure: property relationships. Journal of Chemical Education, Volume 64, Issue 7, 575-581.

[4] Khan, R. S.; Rehman, I. U (2020). Spectrosocopy as a tool for detection and monitoring of Coronavirus (COVID-19). Expert Review of Molecular Diagnostics, DOI: 10.1080/14737159.2020.1766968.

[5] Slater, J. C. (1929). The theory of complex spectra.Physical Review,Volume 34, Issue 10, pp. 1293-1322.

[6] Bhagwan, P. (2005). A hand book of chemical analysis. International Scientific Publishing Academy, pp. 1-289.

[7] Schrodinger, E. (1926). An undulatory theory of the mechanics of atoms and molecules. Physical Review, Volume 28, Issue 6, pp. 1049-1070.

[8] Zumdahl, S. S.; Zumdahl, S. A. (2003). Atomic Structure and Periodicity, In: Chemistry.Boston, MA: Houghton Mifflin Company, pp. 290-294.

[9] Maxwell, J. Clerk. (1865). A Dynamical Theory of the Electromagnetic Field. Philosophical Transactions of the Royal Society of London, Volume 155, Issue 1, pp. 459-512.

[10] De Caro, Cosimo A (2015). UV/VIS Spectrophotometry Fundamentals and Applications. Mettler Toledo,pp. 3-50.

[11] Dingle, H (1935). Applications of Spectroscopy. Journal of the Royal Astronomical Society of Canada, Volume 29, Issue 1, pp. 387-397.

[12] Lakowicz J.R. (2006). Instrumentation for Fluorescence Spectroscopy, In: Principles of Fluorescence Spectroscopy. Springer, Boston, MA, pp. 27-61.

\section{AbBreviations}

- NMR - Nuclear Magnetic Resonance

- EMR - Electromagnetic Radiation

- EMS - Electromagnetic Spectrum

- UV/Vis - Ultraviolet / visible Spectroscopy

- $\mathbf{H}$ - Hydrogen

- C - Carbon

- N - Nitrogen

- P - Phosphorous

- MHz - megahertz

- ATP - Adenosine Triphosphate

- IR - Infrared Spectroscopy 